\section{MS13-P10 Crystal structure of the calcitonin receptor ectodomain in complex with a truncated salmon calcitonin analogue.}

Eva Johansson ${ }^{1}$, Jakob L. Hansen ${ }^{1}$, Ann Maria K. Hansen ${ }^{1}$, Allan C. Shaw ${ }^{1}$, Peter Becker ${ }^{1}$, Lauge Schäffer ${ }^{1}$, Steffen Reedtz-Runge ${ }^{1}$

1. Novo Nordisk A/S

email: evjh@novonordisk.com

Calcitonin (CT) is a peptide hormone consisting of 32 amino acid residues which possesses the ability to lower the calcium plasma concentration. Salmon CT (sCT) has been utilized for 40 years as therapeutic agent for treatment of metabolic bone diseases such as osteoporosis and Paget's disease, due to its high potency which is 40-50 times higher compared to human CT. The calcitonin receptor is a Class $\mathrm{B} G$ protein-coupled receptor (GPCR). Class B GPCRs are characterized by having a large ectodomain (ECD) of 100-160 amino acid residues with a conserved tertiary structure stabilized by three conserved disulfide bridges. The ECD binds the $\mathrm{C}$-terminal region of the peptide ligand and thereby positions the $\mathrm{N}$ - terminal part of the ligand for binding to the transmembrane domain to initiate signaling. The crystal structure of the human $\mathrm{CT}$ receptor ectodomain (CTR ECD) in complex with a truncated analogue of SCT, [BrPhe $\left.{ }^{22}\right] \mathrm{sCT}(8-32) \quad\left(\left[\mathrm{BrPh}^{22}\right]=\right.$ 4-bromo-L-phenylalanine at position 22), has been determined to $2.1 \AA$ resolution. The structure of the CTR ECD is similar to other Class B GPCRs and the ligand binding site is similar to the binding site of the homologous receptors for the calcitonin gene-related peptide (CGRP) and adrenomedulin (AM).

The pharmacology of the CT family of peptides is complex due to heterodimerization of CTR or CT receptor-like receptor (CLR) with a membrane bound receptor activity-modifying protein (RAMP). There are three different variants of RAMPs: RAMP-1, RAMP-2 and RAMP-3, which have $\sim 30 \%$ amino acid sequence identity and the ligand specificity of CTR and CLR is determined by the interaction (or not) with the RAMPs. CTR is on its own the receptor for CT, while in combination with RAMP-1 or RAMP-3 it changes ligand specificity and becomes the amylin receptor. The heterodimer of CLR and RAMP-1 is the receptor for CGRP whereas the heterodimer of CLR and RAMP-2 or RAMP-3 are the receptors for AM.

Interestingly the receptor-bound structure of the truncated SCT ligand differs from the previously determined receptor-bound structures of the homologous ligands CGRP and AM (Booe et al, 2015). They all adopt an extended conformation followed by a C-terminal beta turn, however, the sCT ligand adopts a type II turn (Gly28-Thr31) whereas CGRP and AM adopt type I turns. Our results suggest that a type II turn is the preferred conformation of CT whereas a type I turn is the preferred conformation of peptides that require RAMPs; CGRP, AM and amylin.

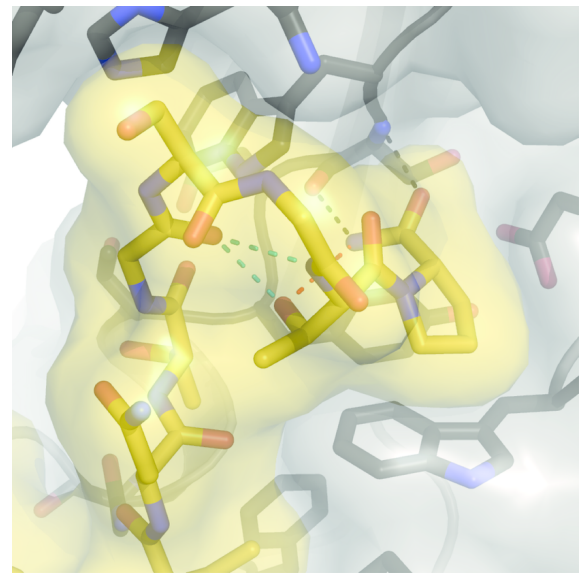

Figure 1. Closeup on the beta turn and the C-terminal prolineamide of $\left[\mathrm{BrPhe}^{22}\right] \mathrm{sCT}(8-32)$ (yellow) and cartoon of CTR ECD (grey) with the side chains of amino acid residues within $4 \AA$ from C-terminal part of $\left[\mathrm{BrPhe}^{22}\right] \mathrm{sCT}(8-32)$ in stick representation. Hydrogen bonds are marked with dotted lines.

Keywords: calcitonin, ectodomain, G protein-coupled receptor 